\title{
The prevalence of subjective frailty and factors associated with frailty in Taiwan
}

\author{
Chin-Ying Chen ${ }^{\mathrm{a}}$, Shwu-Chong $\mathrm{Wu}^{\mathrm{b}}$, Liang-Ju Chen ${ }^{\mathrm{b}}$, Bee-Horng Lue ${ }^{\mathrm{a}, \mathrm{c}, \text { * }}$ \\ ${ }^{a}$ Department of Family Medicine, National Taiwan University Hospital, No. 7 Chung-Shan South Road, Taipei, Taiwan 100 \\ ${ }^{b}$ Institute of Health Policy and Management, College of Public Health, National Taiwan University. Room 621, No. 17 Xu-Zhou Rd., Taipei, Taiwan 100 \\ ${ }^{c}$ Department of Family Medicine, College of Medicine, National Taiwan University, No. 1 Sec. 1, Ren-Ai Road, Taipei, Taiwan 100
}

A R T I C L E I N F O

Keywords:

Frailty of elderly in Taiwan

\begin{abstract}
A B S T R A C T
This study estimated the prevalence of frailty and identified the factors associated with frailty in Taiwan using data from the Survey of Health and Living Status of the Elderly. A nationwide probability sample including 2,238 individuals aged $\geq 65$ years was interviewed in 2003 . Based on the Cardiovascular Health Study conducted by Fried, five phenotypes of frailty were selected: poor appetite, exhaustion, low physical activity, poor walking ability, and poor twisting ability of fingers. Participants were classified as nonfrail, prefrail, and frail if they met 0,1 or 2 , and $\geq 3$ criteria. The prevalences of nonfrailty, prefrailty, and frailty were $55.1 \%, 40.0 \%$, and $4.9 \%$, respectively. The prevalence of frailty increased with age and was greater in women. Frailty was associated with less education, no spouse, disability, higher rates of comorbid chronic diseases, depressive symptoms, and geriatric syndromes. Specific drug use, such as hypnotics, analgesics, herbal drugs, and parenteral fluid supplements was positively associated with frailty. The use of multivitamins, fish oil, and vitamin E was negatively associated with frailty. The prevalence of frailty is lower in Taiwan than in Western countries. Depressive symptoms, geriatric syndromes, and specific medication use are potential fields for frailty prevention in community-dwelling older adults.
\end{abstract}

(c) 2010 Elsevier Ireland Ltd. All rights reserved.

\section{Introduction}

The discovery of effective interventions to prevent or delay disability in older persons is a public health priority. Most likely to benefit from such interventions are frail individuals who are not yet disabled and those with early disability who are at high risk of progression (Ferrucci et al., 2004). Randomized, controlled trials of exercise, comprehensive geriatric assessment (Gill et al., 2002; Reuben, 2002), and interventions targeting multiple risk factors have shown promising early results (Tinetti et al., 1994). The recommendations of the Frailty Working Group focus on preventing progressive disability, not disability caused by acute events such as stroke or hip fracture (Ferrucci et al., 1996). Although progressive disability is common, preventive approaches have rarely been studied. The concept of physical frailty was used to create a working definition of the population at high risk for disability onset or progression (Ferrucci et al., 2004).

Frailty is considered to be highly prevalent with increasing age and to confer a high risk for adverse health outcomes, including mortality, institutionalization, falls, and hospitalization (Speechley and Tinetti, 1991; Winograd, 1991; Rockwood et al., 1999; Espinoza and Walston, 2005; Bandeen-Roche et al., 2006;

\footnotetext{
* Corresponding author. Department of Family Medicine, National

Taiwan University, No. 1 Sec. 1, Ren-Ai Road, Taipei, Taiwan 100.

Tel.: 886-2-23123456, ext: 65348; fax: 886-2-23118674.

E-mail address: grace@ntu.edu.tw (B-H. Lue).
}

Gill et al., 2006). Geriatricians define frailty as a biologic syndrome of decreased reserve and resistance to stressors, resulting from cumulative declines across multiple physiologic systems, and causing vulnerability to adverse outcomes (Fried et al., 2001). This concept distinguishes frailty from disability. Because of the similarity with disability in associated outcomes (with the exception of frailty itself being a cause of disability), and the frequency of the co-occurrence of frailty and disability, there has been much definitional confusion between frailty and disability/dependence. However, there is an increasing consensus that differentiating frailty from disability may improve our understanding of the aging process and offer new opportunities for prevention and care in clinical geriatrics (Fried et al., 2004).

Physical frailty is an abnormal physiological state that can range from mild to severe stages (Ferrucci et al., 2004). Frailty is thought to be present along a continuum of severity (Fried et al., 2005). A physical frail state may be clinically detected before disability, as well as a more advanced state of "clinically overt" physical frailty (Ferrucci et al., 2004). Interventions for those with physical frailty might only target disability prevention, whereas interventions for those with clinically overt physical frailty might target the delay or reversal of disability progression (Ferrucci et al., 2004).

The overall prevalence of frailty in the community-dwelling population of Western countries has been reported to be around 6-20\% using different definitions of frailty (Chin et al., 1999; Fried et al., 2001; Binder et al., 2005; Goggins et al., 2005; Semba et al., 
2006a; Avila-Funes et al., 2008; Fernandez-Bolaños et al., 2008) The prevalence of frailty in Taiwan and the factors associated with frailty have not been established. In order to make a global estimation of the number of frail elders in Taiwan, a retrospective study based on a nationwide survey in Taiwan was conducted.

\section{Subjects and methods}

\subsection{Subjects}

A nationwide Survey of Health and Living Status of the Elderly in Taiwan has been held every 4 years since 1989. The fifth survey was held in 2003. A probability sample with 2,238 individuals aged 65 years and older was interviewed. The detailed research design and sampling strategy have been reported elsewhere (Glei et al., 2005; Zimmer et al., 2005)

\subsection{Methods}

Based on the Cardiovascular Health Study conducted by Fried et al. (2001), five proxy-phenotypes of frailty were selected retrospectively from the fifth survey in 2003.

Weight loss: Because there was no record of weight loss from the fifth survey, we used poor appetite as the substitute. Using the CES-D depression Scale (Boey, 1999), the following two statements were read, "I did not feel like eating, my appetite was poor." The question was asked "How often in the last week did you feel this way?" $0=$ rarely or none of the time ( $<1$ day), $1=$ some or a little of the time (1-2 days), 2 = a moderate amount of the time (3-4 days), or $3=$ most of the time. Subjects answering " 2 " or " 3 " were categorized as frail by the weight loss criterion.

Exhaustion: Using the CES-D Depression Scale (Boey, 1999), the following two statements were read, (a) "I felt that everything I did was an effort." (b) "I could not get going." The question was then asked, "How often in the last week did you feel this way?" $0=$ rarely or none of the time ( $<1$ day), $1=$ some or a little of the time (1-2 days), 2 = a moderate amount of the time (3-4 days), or $3=$ most of the time. Subjects answering " 2 " or " 3 " to either of these questions were categorized as frail by the exhaustion criterion.

Physical activity: Physical activity was measured by either: (a) energy expenditure by the frequency, duration, and types of physical activity; or (b) the sum of the weighted score calculated from the type and frequency of leisure time activity. For (a), the energy consumption of different types of physical activity was defined according to the criteria of the International Physical Activity Questionnaire (IPAQ)-Taiwan edition (Qu and Li, 2004). This variable was stratified by sex. Men with $<383$ Kcal of physical activity per week were considered frail, while women with $<270$ Kcal per week were considered frail. For (b), the weighted score was determined at a consensus meeting. Those who had moderate physical activity daily were assigned a weighted score of 4 . Those who did the same physical activity at a frequency of 1-2 times/week or less than once/week were assigned a weighted score of 2 and 0.8 , respectively. Those who had leisure time activity daily, such as singing, dancing, or Tai-Chi exercise, were assigned a weighted score of 2 . Those who did the same physical activity at a frequency of 1-2 times/week or less than once/week were assigned a weighted score of 1 and 0.4 , respectively. Those who had leisure time activity daily, such as watching TV, reading books, chatting, or fishing, were assigned a weighted score of 1 . Those who did the same activity at a frequency of 1-2 times/week or less than once/week were assigned a weighted score of 0.5 and 0.2 , respectively. Men with a weighted score less than 3 and women with a weighted score less than 2 were categorized as frail.

Walk time: Using the Nagi questionnaire (Nagi, 1976; Nagi and Marsh, 1980), the following statement was read, "Did you have any difficulty in walking 200 to 300 meters?" Subjects answering "yes" to the question were categorized as frail by the walk time criterion.

Grip strength: Using the Nagi questionnaire, the following statement was read, "Did you have any difficulty in picking up or twisting using your fingers?" Subjects answering "yes" to the question were categorized as frail by the grip strength criterion.

Subjects with a critical mass of characteristics, defined as three or more frailty phenotypes, were considered frail. Those with no characteristics were considered robust, whereas those with one or two characteristics were classified as prefrail.

\subsection{Statistical analysis}

The SPSS-10 software package was used (SPSS Inc, Chicago, IL, USA). Chi-square tests were used to test homogeneity, analyses of variance were used to compare characteristics by categories of frailty, and one-way ANOVA was used to test the differences between different levels of frailty.

\section{Results}

The age of the 2,238 subjects (48.85\% female; $51.2 \%$ male) ranged from 65 to 103 years, with a mean age of $73.3 \pm 1.5$ years. Overall, $4.86 \%$ of the cohort was frail, $40 \%$ were prefrail, and $55.14 \%$ were non-frail. The prevalence of frailty increased with each 5-year age group and was up to two-fold higher for women than men for each age group.

Those who were frail were older, more likely to be female, and had less education, lived alone, and had higher rates of comorbid chronic diseases ( $p<0.05$ for each comparison) (Table 1). They also had significantly higher rates of cardiovascular and pulmonary diseases, renal diseases, diabetes mellitus, arthritis, osteoporosis, fractures, cancers, and depressive symptoms (Table 1). There was no significant difference in liver disease. For peptic ulcers and gout, there was a significant difference between the nonfrail and frail groups. For cataracts and cancer, there was a significant difference between the nonfrail and prefrail groups. Notably, 3.7\% of those who were frail had none of these chronic diseases, $11.0 \%$ had just one, and $85.3 \%$ had more than two diseases, including $88.6 \%$ with depressive symptoms, $49.5 \%$ with hypertension, $42.1 \%$ with cataracts, $38.9 \%$ with heart disease, $33.3 \%$ with diabetes, $32.1 \%$ with peptic ulcer, $32.1 \%$ with osteoporosis, and $31.2 \%$ with arthritis. The average number of diseases for the nonfrail, prefrail, and frail groups was $2.03 \pm 1.76,3.08 \pm 2.09$, and $3.79 \pm 2.13$, respectively; the differences among the groups were significant $(p=0.011)$. The prevalence of frailty increased up to threefold in the group with five or more diseases compared to the group with one disease.

Further analysis explored the association between the frailty phenotype and self-reported physical condition. In Table 1, 33.9\% of those who were frail reported difficulty in IADLs, while only $38.5 \%$ of those who were frail had difficulty in ADLs. There was a stepwise increase in disability with increasing frailty status. However, there was only modest concordance between frailty and disability. Of those who were frail or prefrail, $38.4 \%$ had comorbid disease, $9 \%$ had IADL or ADL disability, $38.4 \%$ had both comorbid disease and IADL/ADL disability, and $14.2 \%$ had neither IADL/ADL disability nor comorbidity.

Those who were frail were more likely to have urinary incontinence, stool incontinence, pain, falls (single or recurrent), poor vision, poor hearing, eating difficulty, and mobility impairment (Table 2).

Use of specific drugs (Table 3), such as hypnotics, analgesics, herbal drugs, and parenteral fluid supplements, was positively associated with frailty. However, the use of multivitamins, fish oil, and vitamin E was negatively associated with frailty. Current smokers were less frail. Sedatives and calcium supplements were not associated with frailty. 
Table 1

Demographic and health characteristic distribution of the sample, $\mathrm{n}(\%)$

\begin{tabular}{|c|c|c|c|c|c|}
\hline \multirow{2}{*}{ Variable } & \multicolumn{4}{|l|}{$\mathrm{n}(\%)$} & \multirow[t]{2}{*}{$\mathrm{p}$} \\
\hline & All & Non-frail & Pre-frail & Frail & \\
\hline \multicolumn{6}{|l|}{ Age (years) } \\
\hline $65-69$ & $769(34.4)$ & $518(39.8)$ & $223(29.6)$ & $28(15.3)$ & $<0.001$ \\
\hline $70-74$ & $632(28.2)$ & 389 (29.9) & $209(27.8)$ & $34(18.6)$ & \\
\hline $75-79$ & $455(20.3)$ & 244 (18.7) & $168(22.3)$ & $43(23.5)$ & \\
\hline $80-84$ & 255 (11.4) & $114(8.8)$ & $100(13.3)$ & $41(22.4)$ & \\
\hline $85+$ & $127(5.7)$ & $37(2.8)$ & $53(7.0)$ & $37(20.2)$ & \\
\hline Sex & & & & & $<0.001$ \\
\hline Male & $1,147(51.3)$ & $700(53.8)$ & $373(49.5)$ & $74(40.4)$ & \\
\hline Female & $1,092(48.8)$ & $602(46.2)$ & $380(50.5)$ & $110(60.1)$ & \\
\hline Education & & & & & $<0.001$ \\
\hline Illiteracy & $847(37.8)$ & $422(32.4)$ & $329(43.7)$ & $96(52.5)$ & \\
\hline Elementary & $907(40.5)$ & $563(43.2)$ & $284(37.7)$ & $60(32.8)$ & \\
\hline Junior high & $220(9.8)$ & $134(10.3)$ & $71(9.4)$ & $15(8.2)$ & \\
\hline Senior high + & $264(11.8)$ & $183(14.1)$ & $69(9.2)$ & $12(6.6)$ & \\
\hline Marital status & & & & & $<0.001$ \\
\hline Married & $1,426(63.7)$ & $881(67.7)$ & $459(61.0)$ & $86(47.0)$ & \\
\hline Single, widow & $811(36.2)$ & $421(32.3)$ & $293(38.9)$ & $97(53.0)$ & \\
\hline \multicolumn{6}{|l|}{ Prevalent diseases } \\
\hline CVD & $187(8.4)$ & $42(3.4)$ & $125(14.0)$ & $20(18.5)$ & $<0.001$ \\
\hline Hip fracture & $87(3.9)$ & $20(1.6)$ & $58(6.5)$ & $9(8.3)$ & $<0.001$ \\
\hline Gout & $218(9.7)$ & $101(8.2)$ & $97(10.8)$ & $20(18.3)$ & $<0.001$ \\
\hline Diabetes & $398(17.8)$ & $154(12.5)$ & $208(23.3)$ & $36(33.3)$ & $<0.001$ \\
\hline Renal disease & $211(9.4)$ & $82(6.2)$ & $111(12.4)$ & $18(16.5)$ & $<0.001$ \\
\hline COPD & $312(13.9)$ & $136(11.0)$ & $150(16.8)$ & $26(23.9)$ & $<0.001$ \\
\hline OA spine & $291(13.0)$ & $132(10.7)$ & $135(15.1)$ & $24(22.2)$ & 0.002 \\
\hline Heart disease & $522(23.3)$ & $228(18.5)$ & $252(28.1)$ & $42(38.9)$ & $<0.001$ \\
\hline Osteoporosis & $488(20.0)$ & $185(15.0)$ & $228(25.5)$ & $35(32.1)$ & $<0.001$ \\
\hline Peptic ulcer & $487(21.8)$ & $237(19.2)$ & $215(24.0)$ & $35(32.1)$ & 0.002 \\
\hline Cancer & $76(3.4)$ & $30(2.4)$ & $41(4.6)$ & $5(4.6)$ & 0.02 \\
\hline Arthritis & $521(23.3)$ & $221(17.9)$ & $226(29.7)$ & $34(31.2)$ & $<0.001$ \\
\hline Hypertension & $935(41.8)$ & $455(36.8)$ & $426(47.7)$ & $54(49.5)$ & $<0.001$ \\
\hline Cataract & $788(35.2)$ & $387(31.4)$ & $356(39.6)$ & $45(42.1)$ & 0.004 \\
\hline Hepatobiliary disease & $203(9.9)$ & $101(8.2)$ & $93(10.4)$ & $9(8.3)$ & 0.362 \\
\hline $\begin{array}{l}\text { Depressive symptoms, } \\
\text { CES-D } \geq 10\end{array}$ & $406(19.8)$ & $70(5.8)$ & $243(32.1)$ & $93(88.6)$ & $<0.001$ \\
\hline Number of chronic diseases & & & & & $<0.001$ \\
\hline 0 & $341(15.2)$ & $259(21.0)$ & $78(8.7)$ & $4(3.7)$ & \\
\hline 1 & $475(21.2)$ & $324(26.3)$ & $139(15.5)$ & $12(11.0)$ & \\
\hline 2 & $427(19.1)$ & $231(18.7)$ & $178(19.9)$ & $18(16.5)$ & \\
\hline 3 & $346(15.5)$ & $162(13.1)$ & $166(18.5)$ & $18(16.5)$ & \\
\hline 4 & $280(12.5)$ & $129(10.5)$ & $133(14.9)$ & $18(16.8)$ & \\
\hline $5+$ & $368(16.5)$ & $128(10.4)$ & $201(22.5)$ & $39(35.8)$ & \\
\hline Self-reported disability & & & & & $<0.001$ \\
\hline No disability & $1,679(75.0)$ & $1,150(93.27)$ & $499(55.75)$ & $30(27.6)$ & \\
\hline$\geq 1$ IADL disability & $287(12.9)$ & $69(5.6)$ & $181(20.22)$ & 37 (33.9) & \\
\hline$\geq 1$ ADL disability & $271(12.1)$ & $14(1.13)$ & $215(24.03)$ & $42(38.5)$ & \\
\hline Total & $2,238(100.0)$ & $1,302(100.0)$ & $753(100.0)$ & $183(100.0)$ & \\
\hline
\end{tabular}


Table 2

Geriatric syndromes and frailty among the subjects, $\mathrm{n}(\%)$

\begin{tabular}{llllll}
\hline Variable & $\mathrm{n}(\%)$ & & $\mathrm{p}$ \\
\cline { 2 - 4 } & All & Non-frail & Pre-frail & Frail & \\
\hline Urinary incontinence & $357(16.0)$ & $107(8.7)$ & $215(24.0)$ & $35(32.1)$ & $<0.001$ \\
Stool incontinence & $98(7.9)$ & $27(2.8)$ & $57(13.4)$ & $13(20.2)$ & $<0.001$ \\
Pain & $1,085(52.2)$ & $491(40.7)$ & $500(65.2)$ & $94(87.9)$ & $<0.001$ \\
Fall, once & $817(36.5)$ & $401(32.5)$ & $369(41.2)$ & $47(43.5)$ & 0.001 \\
Falls, $\geq 2$ & $205(9.2)$ & $66(5.3)$ & $110(12.3)$ & $29(26.9)$ & $<0.001$ \\
Poor vision & $437(19.5)$ & $149(12.1)$ & $239(26.7)$ & $49(45.4)$ & $<0.001$ \\
Poor hearing & $318(14.2)$ & $120(9.7)$ & $175(19.5)$ & $23(21.1)$ & $<0.001$ \\
Eating difficulty & $632(28.2)$ & $225(18.2)$ & $347(38.8)$ & $60(55.0)$ & $<0.001$ \\
Mobility impairment & $363(16.2)$ & $30(2.4)$ & $279(31.7)$ & $54(49.5)$ & $<0.001$ \\
Total & $2,238(100.0)$ & $1,302(100.0)$ & $753(100.0)$ & $183(100.0)$ & \\
\hline
\end{tabular}

Table 3

Drugs and frailty among the subjects $(n=2,238)$

\begin{tabular}{llllll}
\hline Variable & $\mathrm{n}(\%)$ & & $\mathrm{p}$ \\
\cline { 2 - 5 } & All & Non-frail & Pre-frail & Frail & \\
\hline Hypnotics & $217(97.7)$ & $86(6.8)$ & $110(12.3)$ & $21(19.3)$ & $<0.001$ \\
Analgesics for arthritis & $341(15.3)$ & $117(9.5)$ & $193(21.6)$ & $31(28.4)$ & $<0.001$ \\
Other analgesics & $149(6.7)$ & $61(5.0)$ & $74(8.3)$ & $14(12.9)$ & $<0.001$ \\
Herbal drugs & $328(14.7)$ & $150(12.2)$ & $149(16.7)$ & $29(26.6)$ & $<0.001$ \\
Parenteral fluid supplements & $72(3.2)$ & $21(0.1)$ & $43(4.8)$ & $8(7.34)$ & $<0.001$ \\
Sedatives & $123(5.5)$ & $56(4.5)$ & $58(6.5)$ & $9(8.6)$ & 0.066 \\
Fish oil & $178(8.0)$ & $118(9.6)$ & $53(5.9)$ & $7(6.4)$ & 0.007 \\
Multivitamins & $407(18.2)$ & $258(20.9)$ & $136(15.2)$ & $13(11.9)$ & 0.001 \\
Vitamin E & $191(8.5)$ & $129(10.5)$ & $60(6.7)$ & $2(1.9)$ & 0.066 \\
Calcium & $411(18.6)$ & $229(18.6)$ & $162(18.1)$ & $20(18.4)$ & 0.965 \\
Total & $2,238(100.0)$ & $1,302(100.0)$ & $753(100.0)$ & $183(100.0)$ & \\
\hline
\end{tabular}

\section{Discussion}

The present study provides global insights into frailty in a population-based sample of Chinese older adults who were neither institutionalized nor end-stage. Frailty has been measured in Chinese populations using a frailty index constructed from different formulas of 39 or 62 variables, but the prevalence of frailty has not been reported (Goggins et al., 2005; Woo et al., 2006; Dupre et al., 2009). There are 2.4 million people in Taiwan ( $10 \%$ of the total population) who are aged 65 years and older. The present study is the first to estimate the prevalence of frailty in Chinese people using the Fried Criteria, and it was lower than previously reported in other populations. This may be due to underestimation from using the proxy phenotypes of Fried's frailty criteria, less population aging, or better health in older Chinese people. Further community-based studies with Fried's frailty criteria are indicated.

The demographic factors associated with frailty were similar to those of past reports: older age, female sex, less education, poor health, and higher rates of chronic comorbidities (Fried et al., 2001; Binder et al., 2005; Goggins et al., 2005; Woo et al., 2006; Avila-Funes et al., 2008; Fernandez-Bolaños et al., 2008; SantosEggimann et al., 2008; Dupre et al., 2009). Regarding specific diseases, liver disease was not associated with frailty. The possible explanation for this is that chronic liver diseases, such as liver cirrhosis, usually involve patients younger than 65 years old (http:// www.doh.gov.tw/ufile/Doc/S02/87-eng.doc). The low prevalence of chronic liver disease among the elderly makes it contribute little to frailty. For peptic ulcers and gout, there was a significant difference only between the nonfrail and frail groups. Peptic ulcer and gout are usually benign diseases that may contribute to frailty if the peptic ulcer results in appetite change and weight loss, or the gout results in polyarthritis and deformity. Peptic ulcer disease's contribution to frailty makes it a remediable factor in the transition to frailty. For cataracts and cancer, there was a significant difference only between the nonfrail and prefrail groups. It is possible that the severely frail cancer patients had a short lifespan, and terminal cancer patients were excluded. The sensory organ diseases (nonsystemic diseases) such as cataracts may result in a prefrail status. The early diagnosis and treatment of cataracts is important for preventing the early stage of frailty.

The present study revealed a high prevalence of depressive symptoms in frail elders. Depression was excluded in Fried's original study (Fried et al., 2001). The association between depression and frailty was reported in elders with cardiometabolic risk factors and heart failure (Kanauchi et al., 2008; Lupón et al., 2008). The causal relationship between frailty and depression is unknown. However, the diagnosis and treatment of depression in frail older persons are potentially remediable factors in frailty prevention.

Comorbidities play an important role in frailty among elderly Chinese (Table 1). Only 3.7\% of those who were frail had none of these chronic diseases, which is lower than the $7.3 \%$ reported by Fried et al (2001), and $86.3 \%$ had more than two diseases, which is higher than the 68\% reported by Fried et al (2001). For the prefrail group, $85.8 \%$ had more than two diseases, which is more than the 53.5\% reported by Fried et al (2001).

Our study demonstrated the relationship between frailty and geriatric syndromes. In addition to depressive symptoms, a higher prevalence of pain (87.9\%), eating difficulty (55.0\%), and mobility impairment (49.5\%) in frail elderly revealed the basic components 
of frailty. The high association between pain and frailty is obvious (Blyth et al., 2008). Pain occurs within a constellation of agingrelated symptoms that are harbingers of declining health and functioning. Pain and analgesic use are the two sides of a symptom. The early identification of pain and analgesic use offer other potential remediable factors in frailty prevention. Poor nutrition and mobility statuses are cardinal components in frailty, and their early identification in primary care is important for the prevention of frailty.

It has been reported that the use of specific drugs, such as digitalis, diuretics, other cardiovascular drugs, hypnotics, sedatives, analgesics, and laxatives, was related to impaired quality of life (Jensen et al., 1994). However, this is the first report of specific drugs, such as hypnotics, analgesics, herbal drugs, and parenteral fluid supplements, being positively associated with frailty. The association of hypnotics with frailty may be due to the resulting lack of balance. The association of analgesics with frailty may be due to the disease itself or due to symptomatic pain. Parenteral fluid supplements and herbal medicine are Chinese cultural health behaviors. Weakness, loss of energy, and malnutrition are the common causes of seeking parenteral fluid supplements. Health beliefs of chronic illness, especially with nonresolving symptoms or medically incurable diseases, are the common reasons for herbal medicine. It is necessary to have a comprehensive assessment when dealing with patients who are taking such medications in order to prevent frailty.

Regarding other medications, the use of multivitamins, fish oil, and vitamin E was negatively associated with frailty. This finding supports previous studies that showed that low micronutrients predict frailty (Michelon, 2006; Semba et al., 2006b; Matteini et al., 2008), which is a potential intervention to prevent frailty.

\section{Conclusion}

The global estimate of the prevalence of frailty in Taiwan using the retrospective proxy Fried criteria was lower than that in Western countries. Further community studies with the Fried criteria are indicated. Depressive symptoms, other geriatric syndromes, and specific medication use are potential fields for frailty prevention in community-dwelling older adults.

\section{Conflict of interest statement}

None

\section{References}

Analysis of the Leading Causes of Death in Taiwan in 1998. http://www.doh.gov.tw/ ufile/Doc/S02/87-eng.doc

Avila-Funes, J.A., Helmer, C., Amieva, H., Barberger-Gateau, P., Le Goff, M., Ritchie, K., Portet, F., Carrière, I., Tavernier, B., Gutiérrez-Robledo, L.M., Dartigues, J.F., 2008. Frailty among community-dwelling elderly people in France: the three-city study. J. Gerontol. A: Biol. Sci. Med. Sci. 63, 1089-1096.

Bandeen-Roche, K., Xue, Q.L., Ferrucci, L., Walston, J., Guralnik, J.M., Chaves, P., Zeger, S.L., Fried, L.P., 2006. Phenotype of Frailty: Characterization in the Women's Health and Aging Studies. J. Gerontol. A: Biol. Sci. Med. Sci. 61, 262-266.

Binder, E.F., Yarasheski, K.E., Steger-May, K., Sinacore, D.R., Brown, M., Schechtman, K.B., Holloszy, J.O., 2005. Effects of progressive resistance training on body composition in frail older adults: results of a randomized, controlled trial. J. Gerontol. A: Biol. Sci. Med. Sci. 60, 1425-1431.

Blyth, M., Rochat, S., Cumming, R.G., Creasey, H., Handelsman, D., Couteur, D., Naganathan, V., Sambrook, P., Seibel, M., Waite, L. 2008. Pain, Frailty and Comorbidity in Older Men: The CHAMP Study. Pain 140, 224-230.

Boey, K.M., 1999. Cross-validation of a short form of the CES-D in Chinese elderly. Int. J. Geriat. Psychiatry. 14, 608-617.

Chin, A., Paw, M.J., Dekker, J.M., Feskens, E.J., Shouten, E.G., Kromhour, D., 1999. How to select a frail elderly population? A comparison of three working definitions. J. Clin. Epidemiol. 52, 1015-1021.

Dupre, M.E., Gu, D., Warner, D.F., Yi, Z., 2009. Frailty and type of death among older adults in China: prospective cohort study. Br. Med. J. 338, b1175.

Espinoza, S., Walston, J.D., 2005. Frailty in older adults: insights and interventions. Cleveland Clinic J. Med. 72, 1105-1012.
Fernandez-Bolaños, M., Otero, A., Zunzunegui, M.V., Beland. F., Alarcón, T., de Hoyos, C., Castell, M.V., 2008. Sex differences in the prevalence of frailty in a population aged 75 and older in Spain. J. Am. Geriatr. Soc. 56, 2370-2371.

Ferrucci, L., Guralnik, J.M., Simonsick, E., 1996. Progressive versus catastrophic disability. A longitudinal view of the disablement process. J. Gerontol. A: Biol. Sci. Med. Sci. 51, M123-M130.

Ferrucci, L., Guralnik, J.M., Studenski, S., Fried, L.P., Cutler, G.B.Jr., Walston, J.D.; Interventions on Frailty Working Group. 2004. Designing randomized controlled trials aimed at preventing or delaying functional decline and disability in frail, older persons: a consensus report. J. Am. Geriatr. Soc. 52, 625-634.

Fried, L.P., Tangen, C.M., Walston, J., Newmanc, A.B., Hirschd, C., Gottdienere, J. Seemanf, T., Tracyg, R., Koph, W.J., Burkei, G., McBurnie, M.A., 2001. Frailty in older adults: evidence for a phenotype. J. Gerontol. A: Biol. Sci. Med. Sci. 56, M146M156.

Fried, L.P., Ferrucci, L., Darer, J., Williamson, J.D., Anderson, G., 2004. Untangling the concepts of disability, frailty, and comorbidity: implications for improved targeting and care. J. Gerontol. A: Biol. Sci. Med. Sci. 59, M255-M263.

Fried, L.P., Hadley, E.C., Waltson, J.D., Newman, A.B., Guralnik, J.M., Studenski, S., Harris, T.B., Ershler, W.B., Ferrucci, L., 2005. From bedside to bench: research agenda for frailty. Sci. Aging Knowl. Environ. 31, 24-38.

Gill, T.M., Baker, D.I., Gottschalk, M., Peduzzi, P.N., Allore, H., Byers, A., 2002. A program to prevent functional decline in physically frail, elderly persons who live at home. N. Engl. J. Med. 347, 1068-1074.

Gill, T.M., Gahbauer, E.A., Allore, H.G., Ham, L., 2006. Transitions between frailty states among community-living older persons. Arch. Intern. Med. 166, 418-423.

Glei, D.A., Landau, D.A., Goldman, N., Chuang, Y.L., Rodriguez, G., Weinstein, M., 2005. Participating in social activities helps preserve cognitive function: an analysis of a longitudinal, population-based study of the elderly. Int. J. Epidemiol. 34, 864871.

Goggins, W.B., Woo, J., Sham, A., Ho, S.C., 2005. Frailty index as a measure of biological age in a Chinese population. J. Gerontol. A: Biol. Sci. Med. Sci. 60, M1046-M1051. http://www.doh.gov.tw/ufile/Doc/S02/87-eng.doc

Jensen, E., Dehlin, O., Hagberg, B., Samuelsson, G., Svensson, T., 1994. Medical psychological, and sociological aspects of drug treatment in 80-year-olds. Z. Gerontol. 27, 140-144.

Kanauchi, M., Kubo, A., Kanauchi, K., Saito, Y., 2008. Frailty, health-related quality of life and mental well-being in older adults with cardiometabolic risk factors. Int. J. Clin. Pract. 62, 1447-1451.

Lupón, J., González, B., Santaeugenia, S., Altimira, S., Urrutiaa, A., Mása, D., Díeza, C., Pascuala, T., Canoa, L., Vallea, V., 2008. Prognostic implication of frailty and depressive symptoms in an outpatient population with heart failure. Rev. Esp. Cardiol. 61, 835-842.

Matteini, A.M., Walston, J.D., Fallin, M.D., Bandeen-Roche, K., Kao, W.H., Semba, R.D., Allen, R.H., Guralnik, J., Fried, L.P., Stabler, S.P., 2008. Markers of B-vitamin deficiency and frailty in older women. J. Nutr. Health. Aging 12, 303-308.

Michelon, E., 2006. Vitamin and carotenoid status in older women: associations with the frailty syndrome. J. Gerontol. A: Biol. Sci. Med. Sci. 61, M600-M607.

Nagi, S.Z., 1976. An epidemiology of disability among adults in the United States. Milbank Memorial Fund Quarterly 54, 439-467.

Nagi, S.Z., Marsh, J., 1980. Disability, health status, and utilization of health services. Int. J. Health. Serv. 10, 657-676.

Qu, N.N., Li, K.J., 2004. Study on the reliability and validity of international physical activity questionnaire (Chinese Version, IPAQ). Zhonghua Liu Xing Bing Xue Za Zhi. 25, 265-268 (in Chinese).

Reuben, D.B., 2002. Organizational interventions to improve health outcomes of older persons. Med. Care. 40, 416-428.

Rockwood, K., Stadynk, K., MacKnight, C., McDowell, I., Hebert, R., Hogan, D.B., 1999. A brief clinical instrument to classify frailty in elderly people. Lancet 353, 205206.

Santos-Eggimann, B., Cuenoud, P., Spagnoli, J., Junod, J., 2008. Prevalence of frailty in middle-aged and older community-dwelling Europeans living in 10 countries. J. Gerontol. A: Biol. Sci. Med. Sci. 64, M675-M681.

Semba, R.D., Bartali, B., Zhou, J., Blaum, C., Ko, C.W., Fried, L.P., 2006a. Low serum micronutrient concentrations predict frailty among older women living in the community. J. Gerontol. A: Biol. Sci. Med Sci. 61, M594-M599.

Semba, R.D., Blaum, C.S., Bartali, B., Xue, Q.L., Ricks, M.O., Guralnik, J.M., Fried, L.P., 2006b. Denture use, malnutrition, frailty, and mortality among older women living in the community. J. Nutr. Health Aging 10, 161-167.

Speechley, M., Tinetti, M., 1991. Falls and injuries in frail and vigorous community elderly persons. J. Am. Geriatr. Soc. 39, 46-52.

Tinetti, M.E., Baker, D.I., McAvay, G., Claus, E.B., Garrett, P., Gottschalk, M., Koch, M.L., Trainor, K., Horwitz, R.I., 1994. A multifactorial intervention to reduce the risk of falling among elderly people living in the community. N. Engl. J. Med. 331, 821-827.

Winograd, C.H., 1991. Targeting strategies: an overview of criteria and outcomes. J. Am. Geriat. Soc. 39S, 25S-35S.

Woo, J., Goggins, W., Sham, A., Ho, S.C., 2006. Public health significance of the frailty index. Disabil. Rehabil. 28, 515-521.

Zimmer, Z., Martin, L.G., Lin, H.S., 2005. Determinants of old-age mortality in Taiwan. Soc. Sci. Med. 60, 457-470. 